\title{
Risiken, Fehler und Patientensicherheit Risques, erreurs médicales et sécurité des patients
}

Donnerstag, 25. Januar 2001

Jeudi, 25 janvier 2001

08.30-17.00 Uhr / heure

Hörsaal 2 Chirurgie, Inselspital, Bern

Auditoire 2 chirurgie, Hôpital de l'lle, Berne

Anmeldung/inscription:

FMH

Elfenstrasse 18

CH-3000 Bern 16

Tel.: 0313591111

Fax:0313591112

E-mail:fmh@hin.ch

\author{
Referenten / Orateurs \\ Dr. med. H. H. Brunner, Präsident FMH, Bern \\ Dr. med. M. Giger, Mitglied des ZV der FMH, \\ Winterthur \\ Prof. Dr. med. D. Hatch, General Medical Council, \\ London \\ Fürsprecher Hp. Kuhn, FMH, Bern \\ Prof. Dr. med. G. Ollenschläger, AZQ, Köln \\ PD Dr. med. C. Rageth, Chefarzt Frauenklinik, \\ Limmattalspital, Schlieren \\ Prof. Dr. med. M. Stäubli, Chefarzt Medizin, \\ Spital Zollikerberg \\ Dr. med. MHA J. von Overbeck, Chefarzt Swiss-Re, \\ Zürich
}

\section{Programm / programme}

Tagungssprachen: deutsch/französisch/englisch (ohne Übersetzung) Langues: allemand/français/anglais (sans traduction)

\begin{tabular}{|c|c|}
\hline $08.30 \mathrm{Uhr}$ & Empfang / Accueil \\
\hline \multirow[t]{2}{*}{ 09.00 Uhr } & $\begin{array}{l}\text { Eröffnung der Tagung / Ouverture } \\
\text { H.H. Brunner, Präsident FMH }\end{array}$ \\
\hline & Problembeschreibung, Ziel und Ablauf der Tagung \\
\hline $09.30 \mathrm{Uhr}$ & $\begin{array}{l}\text { Begriffe: Risiko, Unsicherheit, Fehler } \\
\text { J. von Overbeck, Zürich }\end{array}$ \\
\hline $09.50 \mathrm{Uhr}$ & $\begin{array}{l}\text { Juristische Aspekte } \\
\text { Hp. Kuhn, Bern }\end{array}$ \\
\hline $10.20 \mathrm{Uhr}$ & Diskussion / Discussion \\
\hline \multirow[t]{2}{*}{$10.30 \mathrm{Uhr}$} & Kaffeepause / Pause café \\
\hline & Vorsitz: H.H. Brunner, Bern \\
\hline $10.45 \mathrm{Uhr}$ & $\begin{array}{l}\text { Experience from British General Council (GMC): incidence and admittance of errors } \\
\text { D. Hatch, GMC, London }\end{array}$ \\
\hline $11.30 \mathrm{Uhr}$ & $\begin{array}{l}\text { Schweizer Studie der Komplikationen in der Inneren Medizin } \\
\text { M. Stäubli, Zollikerberg }\end{array}$ \\
\hline $12.00 \mathrm{Uhr}$ & $\begin{array}{l}\text { Die Problematik der Fehler und der Patientensicherheit in Deutschland } \\
\text { G. Ollenschläger }\end{array}$ \\
\hline $12.30 \mathrm{Uhr}$ & Diskussion / Discussion \\
\hline \multirow[t]{2}{*}{$13.00 \mathrm{Uhr}$} & Lunch / Repas \\
\hline & Vorsitz: M. Stäubli, Zollikerberg \\
\hline $13.45 \mathrm{Uhr}$ & $\begin{array}{l}\text { Was lernt man über Komplikationen und Fehler aus den Datenbanken der ASF und AQC? } \\
\text { C. Rageth, Schlieren }\end{array}$ \\
\hline $14.15 \mathrm{Uhr}$ & $\begin{array}{l}\text { Fehler in der Pharmakotherapie und mögliche Vorbeugungsmassnahmen } \\
\text { M. Giger }\end{array}$ \\
\hline $14.45 \mathrm{Uhr}$ & $\begin{array}{l}\text { GMC programmes for reduction of errors and protection of patients } \\
\text { D. Hatch, GMC, London }\end{array}$ \\
\hline $15.15 \mathrm{Uhr}$ & $\begin{array}{l}\text { Forumdiskussion "Verbesserungsvorschläge» (mit kurzen Stellungnahmen von Interessierten } \\
\text { aus dem Gesundheitswesen und der Öffentlichkeit) } \\
\text { Leitung: H. H. Brunner }\end{array}$ \\
\hline $16.15 \mathrm{Uhr}$ & $\begin{array}{l}\text { Schlussfolgerung und Ausblick } \\
\text { H. H. Brunner }\end{array}$ \\
\hline $16.30 \mathrm{Uhr}$ & Schluss der Tagung / Fin du séminaire \\
\hline
\end{tabular}

